\title{
Anabases
}

ANABASES Traditions et réceptions de l'Antiquité

$15 \mid 2012$

Varia

\section{Jules Toutain et l'éloge de la colonisation}

\section{Aurélie Rodes}

\section{OpenEdition}

Journals

Édition électronique

URL : http://journals.openedition.org/anabases/3675

DOI : 10.4000/anabases.3675

ISSN : 2256-9421

\section{Éditeur}

E.R.A.S.M.E.

\section{Édition imprimée}

Date de publication : 1 avril 2012

Pagination : 59-69

ISSN : 1774-4296

\section{Référence électronique}

Aurélie Rodes, « Jules Toutain et l'éloge de la colonisation», Anabases [En ligne], 15 | 2012, mis en ligne le 01 avril 2015, consulté le 20 octobre 2019. URL : http://journals.openedition.org/anabases/3675 ; DOI : 10.4000/anabases.3675 
Anabases 15 (2012), p. 59-69

\section{Jules Toutain et l'éloge de la colonisation}

Aurélie Rodes

Parmi Les nOms des antiquisants français du $\mathrm{xx}^{\mathrm{e}}$ siècle, celui de Jules Toutain (18651961) n'est pas très connu. Cet archéologue a pourtant dirigé pendant près d'un demi-siècle le prestigieux site d'Alise-Sainte-Reine, célèbre pour avoir été le théâtre de l'affrontement entre Jules César et Vercingétorix en 52 avant J.-C. Il a aussi rédigé avec Désiré Blanchet de nombreux manuels scolaires réputés ${ }^{1}$ et fut un auteur prolixe, exprimant son opinion dans des revues scientifiques.

Plusieurs études récentes se sont cependant intéressées à lui, comme celle de l'équipe Érasme ${ }^{2}$ sur les contributeurs du Dictionnaire des antiquités grecques et romai$n e s^{3}$ ou celle de Clémentine Gutron ${ }^{4}$ portant sur ses travaux archéologiques en Tunisie ou encore celle de Sarah Rey ${ }^{5}$ consacrée aux membres de l'École française de Rome.

1 Jules Toutain est le coauteur, entre 1888 et 1931, d'une quarantaine de titres de manuels scolaires, sans tenir compte des nombreuses rééditions. Ces manuels concernent tous les niveaux scolaires et ont été adaptés à chaque modification de programmes. Ils s'adressent tant aux élèves de l'école primaire qu'à ceux de collège et lycée. Il a aussi conçu des cours destinés aux futures institutrices de l'École normale supérieure de Fontenay-aux-Roses, où il était enseignant.

2 A. Andurand, J. Cazenave et S. Delmas, "Appréhender le Dictionnaire des antiquités grecques et romaines par sa table d'auteurs. Les données statistiques comme outil d'analyse ", Anabases. Traditions et réceptions de l'Antiquité 4 (2006), p. 219-224.

3 Ch. Daremberg, Ed. Saglio et Ed. Pottier, Dictionnaire des antiquités grecques et romaines, Paris, Hachette, 10 vol., 1873-1919.

4 C. Gutron, L'archéologie en Tunisie (XIX'-XXe siècles). Jeux généalogiques sur l'Antiquité, Paris, Karthala, 2010, 327 p.

5 S. ReY, Comment on a écrit l'histoire ancienne à l'École Française de Rome (1873-1940), thèse de doctorat soutenue à l'université de Toulouse II-Le Mirail, sous la direction de Corinne Bonnet et Pascal Payen, 2009, 482 p. 
Son activité d'auteur de manuel a aussi été soulignée ${ }^{6}$ et doit maintenant être mise en relation avec sa conception de la colonisation de l'Afrique du Nord, notamment en observant les liens qu'il a tissés d'une part avec l'Antiquité et d'autre part entre les savoirs universitaires et les contenus de l'enseignement secondaire.

Il n'est point surprenant qu'à la fin du XIX et au début du XXe siècle, Jules Toutain ait fait l'éloge de la colonisation, à une époque où l'intérêt de cette entreprise de l'État français en Afrique du Nord était peu débattu. Les parallèles entre la colonisation, à laquelle il contribue en s'impliquant dans la mise en place de l'archéologie tunisienne, et la colonisation antique sont plus originaux. Afin de mieux cerner ses positions dans ce domaine, il convient tout d'abord d'indiquer comment les rapprochements entre la conquête romaine et celle, moderne, de l'Afrique ont permis à Jules Toutain de faire l'éloge de la colonisation française. On s'intéressera ensuite à la façon dont il a présenté la colonisation antique dans l'enseignement primaire et secondaire afin d'évaluer enfin l'impact d'un tel enseignement.

\section{Jules Toutain ou l'éloge de la colonisation française par le biais de l'expérience romaine}

Jules Toutain a le profil type d'un antiquisant de son temps. Formé à l'École Nationale Supérieure où il a préparé l'agrégation, il poursuit ses recherches à l'École française de Rome avant de s'impliquer dans l'archéologie en Tunisie. Il se place ainsi à la croisée de plusieurs réseaux institutionnels qui lui permettent de devenir maitre de conférences à la faculté des lettres de Caen, puis directeur d'études à l'École pratique des hautes études à la section des sciences religieuses et de prendre la direction des fouilles d'Alésia de 1906 à $1958^{7}$.

6 A. Rodes, « De la chaire à l'école : le rôle des universitaires Antiquisants dans les programmes et les manuels de l'enseignement secondaire au Xxe siècle ", in C. Bonnet, V. KRINGS et C. Valenti (dir.), Connaître l'Antiquité : individus, réseaux, stratégies du XVIII au XXI siècle, Rennes, Presses Universitaires de Rennes, 2010, p. 153-168.

7 Ces travaux archéologiques ont donné lieu à de nombreuses publications dont quatre ouvrages parus dans les années 1930 : J. ToutAin, Les Fouilles d'Alésia et l'ethnographie gallo-romaine, Paris, E. Leroux, 1931, 192 p. ; ID., La Gaule antique vue dans Alésia, La Charité-sur-Loire, A. Delayance, 1932, 231 p. ; ID., Alésia gallo-romaine et chrétienne, La Charité-sur-Loire, A. Delayance, 1933, 200 p. et ID., Le passé et la découverte d'Alésia, La Charité-sur-Loire, A. Delayance, 1948, 48 p. Il a publié également régulièrement des rapports de fouilles dans la revue Pro Alésia et des articles portant sur des découvertes ponctuelles. Certains abordent aussi la portée des événements qui ont eu lieu à Alésia comme l'article J. TOUTAIN, "Les conséquences profondes et les vrais résultats historiques de la prise d'Alésia ", in Pro Alesia, nouvelle série, 25 (1920), p. 97-117. Il a aussi exprimé son point de vue dans une revue destinée à un public différent la Revue internationale de l'enseignement: J. ToutAIN, "La résurrection d'Alésia ", Revue internationale de 
L'originalité de son parcours tient au sujet de sa thèse ${ }^{8}$ qui porte sur la Tunisie, alors sous protectorat français. Tout en s'intéressant à la colonisation romaine de l'Afrique du Nord, il participe à l'administration coloniale en Tunisie et plus particulièrement à la mise en place d'un service des Antiquités. Il est donc bien placé pour relever les analogies entre l'Antiquité et la période contemporaine ${ }^{9}$, soulignées dans la Revue internationale de l'enseignement en 1896 dans un compte rendu portant sur sa thèse :

"Pourquoi le même pays si riche autrefois est-il à présent si pauvre ? [...] Comment les Romains ont-ils résolu le problème de la colonisation ? Nous qui sommes leurs héritiers et leurs continuateurs n'avons-nous rien à apprendre de leur école ? Ce qu'ils ont fait ne peut-il se refaire ? Et par quels moyens ? C'est à ces questions que M. Toutain répond dans son essai [...]. Son livre est celui d'un savant et d'un archéologue, c'est aussi un livre d'actualité : l'expérience des peuples peut bien être aussi utile que celle des individus. [...] Et toujours, M. Toutain nous montre dans le passé un exemple à méditer, une expérience dont il est bon de profiter. Ces sortes de comparaisons s'imposent à l'esprit du lecteur, et l'on sent que l'auteur les a toujours présentes, même s'il ne les formule pas. Ces préoccupations du temps présent sont un attrait de plus, elles captivent au même degré que le travail de savante reconstruction auquel se livre M. Toutain. [...] resterait à souhaiter qu'elle [son étude] ${ }^{10}$ fût connue toujours de nos administrateurs africains ${ }^{11}$. "

Selon ce rapporteur les intentions de Jules Toutain ne sont par clairement formulées; il recommande cependant l'ouvrage aux acteurs de la colonisation contemporaine. Quelques années plus tôt, en 1892, Jules Toutain avait lui aussi présenté l'intérêt des comparaisons entre la colonisation romaine et la colonisation de l'Algérie :

" [...] la conclusion de M. Cat nous paraît fort juste. "Les Romains, dit-il, paraissent avoir assimilé les indigènes beaucoup plus que nous, Français, nous n'avons pu le faire jusqu'ici”. [...] Dans plusieurs passages de son livre, M. Cat a comparé ainsi l'œuvre des Romains en Algérie à celle que nous y accomplissons maintenant. Il faut le féliciter vivement d'avoir compris que souvent les études d'archéologie et d'histoire ancienne ne sont pas purement spéculatives. Malgré la différence des temps et des conditions, les Français

l'enseignement 78 (1924), p. 321-327 ou ID., « Le véritable caractère de la portée historique des Fouilles d'Alésia. Communication présentée à la réunion solennelle de la Société des sciences de Semur, 4/9/1938 tenue à Alise-Sainte-Reine ", Revue internationale de l'enseignement 92 (1938), p. 207-221. Selon lui, à Alésia, les Gaulois n'ont pas perdu puisque la conquête romaine a évité l'invasion germanique.

8 J. Toutain, Les cités romaines de la Tunisie. Essai sur l'histoire de la colonisation romaine dans l'Afrique du Nord, Paris, Thorin A. Fontemoing, 1896, 412 p.

9 J. Toutain, La Tunisie romaine et l'Afrique française : Conférence faite à Tunis, sous le patronage de l'Alliance Française, Les Sables d'Olonne, Imprimerie-Librairie Veuve Jourdan, 1892, $30 \mathrm{p}$.

10 Les mots entre crochets ont été ajoutés pour rendre la compréhension de la phrase d'origine.

11 S. Rocheblave, Revue internationale de l'enseignement 31 (1896), p. 412. 
ne peuvent que tirer profit des méthodes de colonisation qui ont été employées il y a 1600 ans en Numidie et en Maurétanie. Il faut donc s'appliquer à rechercher quelles sont ces méthodes, comment le pays fut organisé, comment toutes les ressources naturelles furent utilisées, comment la population primitive fut assimilée ${ }^{12}$."

Il présentait alors clairement la colonisation antique comme un exemple à suivre tant pour l'organisation administrative et politique qu'en matière d'assimilation des indigènes. Il revint en 1907 sur la question de l'assimilation religieuse en introduction de l'un de ses principaux ouvrages portant sur les cultes païens :

"Mais nous est-il interdit de penser que des questions analogues se posent de nos jours et se poseront encore demain ? Si nous faisons abstraction des circonstances de temps et de lieu, ne peut-on pas voir dans le sujet que nous avons choisi un cas particulier du problème général suivant : comment un peuple vainqueur ou colonisateur, ayant sa religion à lui, doit-il traiter d'autres peuples qui ont également leurs religions, lorsqu'il les a vaincus, soumis, annexés à son empire ou colonisés ? Quelle doit être d'autre part l'attitude de ces peuples à l'égard de la religion du vainqueur ? [...] La politique religieuse [comme celle de Rome] appliquée à ces provinces par le gouvernement impérial peut donc fournir d'utiles indications aux peuples colonisateurs de notre temps. Peut-être y a-t-il là un exemple à étudier et à méditer ${ }^{13}$."

Bien que ce type de remarque ne figure que dans l'introduction de l'ouvrage, l'exemple antique est ici magnifié par un spécialiste de l'histoire romaine qui suggère que la Rome impériale est susceptible de servir de modèle à la $\mathrm{III}^{\mathrm{e}}$ République en matière de colonisation. Ici, seul le point de vue du colonisateur est pris en compte, sans le moindre égard pour la culture africaine précoloniale. Ceci apparaît encore plus nettement dans un article publié en 1894 dans la Revue internationale de l'enseignement sur l'histoire ancienne, où il montre la spécificité de cette période et de ses méthodes :

" [...] nous devons nous efforcer de comprendre et de savoir et de comprendre pourquoi et comment, à l'heure où toutes les nations éprouvent l'impérieux besoin, les unes de créer, les autres d'étendre leur empire colonial ; nous devons le faire, surtout lorsque nous succédons aux peuples anciens dans des régions qui ont été abandonnées à elles-mêmes depuis l'antiquité, qui sont devenues stériles et désertes, et auxquelles nous voulons rendre aujourd'hui toutes leurs richesses d'autrefois ${ }^{14}$."

Il s'agit ici encore d'une lecture très partiale de l'histoire. Jules Toutain oblitère toute l'époque arabo-musulmane et sa brillante civilisation qui est loin d'avoir rendu "stériles et désertes " les régions du Maghreb. Pourtant les positions de Jules Toutain

12 J. Toutain, "Chronique ", Mélanges d'archéologie et d'histoire 12 (1892), p. 204.

13 J. Toutain, Les cultes païens dans l'Empire romain, Paris, E. Leroux, 1907, p. 14-15.

14 J. ToutAIN, "Sur l'histoire ancienne ", Revue internationale de l'enseignement 28 (1894), p. 137. 
ne suscitèrent, semble-t-il, aucune réaction, sans doute parce qu'elles étaient dans l'air du temps. Les récents travaux sur les usages du passé, comme ceux de Giovanni Lévi, montrent que notre historien ne fait en effet pas exception :

"Ainsi, l'histoire a toujours produit des visions du monde qui comportaient, indissociablement, un élément politique dont l'usage, conscient ou inconscient, était de toute façon inévitable. Cet usage a lui-même une histoire qu'il vaut la peine d'interroger, histoire faite d'éléments nombreux, au premier rang desquels figure la relation entre l'historien et un public de lecteurs qui a changé avec le temps ${ }^{15}$."

Jules Toutain n'a sans doute pas plus que les autres intellectuels de son temps fait l'éloge de la colonisation antique, ni davantage utilisé le passé pour éclairer le présent. L'effet d'accumulation perceptible à la lecture des extraits sélectionnés doit être relativisé : les travaux de Jules Toutain portent essentiellement sur l'Antiquité. Ayant été amené à rédiger des manuels abordant l'histoire ancienne comme l'histoire contemporaine, les liens entre ces deux périodes lui ont sans doute semblé plus évidents. Il convient donc d'envisager maintenant comment ses idées ont été introduites dans l'enseignement.

\section{Les analogies entre colonisation antique et colonisation contemporaine dans l'enseignement secondaire}

Jules Toutain manifeste un intérêt prononcé pour la vulgarisation des acquis de la recherche, que ce soit en participant au Dictionnaire des antiquités grecques et romaines ${ }^{16}$ ou en collaborant très activement à la revue Pro Alesia, dont l'objectif était de faire état des découvertes effectuées sur le site d'Alésia, ou encore en proposant des articles concernant aussi bien l'histoire ancienne que les rythmes scolaires ${ }^{17}$ dans la Revue internationale de l'enseignement. Il indiquait ainsi dans l'un de ces articles :

" [...] En outre, il faut aborder ces études dans un esprit à la fois scientifique et didactique : scientifique par la méthode que l'on appliquera, méthode analytique et synthétique ;

15 G. LÉvI, «Le passé lointain sur l'usage politique de l'histoire ", in F. HarTOG et J. Revel (dir.), Les usages politiques du passé, Paris, Éditions de l'École des hautes études en sciences sociales, 2001, p. 26.

16 Ch. Daremberg, Ed. Saglio et Ed. Pottier, Dictionnaire des antiquités. Jules Toutain intervient entre 1900 et 1915 sur le volume « L-M » du dictionnaire, avec quinze notices consacrées aux divinités romaines ou italiques, selon J. CAZENAVE «Le "mystère Toutain" à la lumière de sa contribution au Dictionnaire des antiquités grecques et romaines ", Anabases. Traditions et réceptions de l'Antiquité 4 (2006), p. 197-203.

17 J. ToutAIn, "L'enseignement secondaire ", Revue internationale de l'enseignement 56 (1908), p. 235 et ID., "À propos du surmenage scolaire. Une simple comparaison ", Revue internationale de l'enseignement 84 (1930), p. 302. 
didactique par les conclusions que l'on formulera, par les enseignements que l'on tirera des vicissitudes de l'humanité ${ }^{18}$."

Le souci de diffuser ses recherches dans l'enseignement se concrétise rapidement puisque cet universitaire se consacre de 1888 à 1931 à la rédaction de nombreux manuels scolaires de l'enseignement primaire et secondaire. Les manuels sont des témoins particulièrement intéressants pour l'étude de la réception de l'histoire ${ }^{19}$. Certains d'entre eux retiennent particulièrement l'attention aujourd'hui car ils ont dressé des parallèles entre les Gaulois et les populations du Maghreb. Ainsi, un manuel rédigé par deux éminents spécialistes de l'histoire romaine, Paul Guiraud et Georges Lacour-Gayet, qui ont eux aussi participé au Dictionnaire des antiquités grecques et romaines, proposait-il en 1885 une comparaison avec les populations colonisées pour décrire les Gaulois :

«Leur aspect est effrayant [...]. Leurs maisons furent d'abord les grottes naturelles ou les gourbis de nos populations algériennes, des huttes rondes de branchages recouvertes de terre pétrie ou gazonnée, avec un trou au sommet pour la fumée ${ }^{20}$."

Soulignons l'usage du possessif pour parler des populations algériennes et le terme pour le moins désobligeant pour évoquer l'habitat algérien. Il paraît peu probable que les élèves de quatrième de l'époque aient eu la moindre idée de ce à quoi ressemblait l'habitat algérien, ce qui rend la comparaison pédagogiquement peu intéressante. La remarque vaut aussi pour les célèbres manuels édités chez Hachette, dans la collection Malet-Isaac, qui font appel aux peuples colonisés d'Afrique du Nord pour faire comprendre qui étaient les Gaulois :

"La masse du peuple se composait de paysans : on peut se représenter ces paysans gaulois comme ceux des tribus montagnardes au Maroc, un peu agriculteurs, un peu pasteurs, grands chasseurs, tous armés ${ }^{21}$."

Ce manuel donne lui aussi une curieuse image des paysans d'Afrique du Nord " tous armés ". L'assimilation entre colonisés et Gaulois n’est au demeurant pas spécifique aux manuels de collège. Dominique Maingueneau, qui a étudié ceux de l'enseignement élémentaire, propose des conclusions similaires :

18 TOUTAin, "Sur l'histoire ancienne », p. 138.

19 Alain Corbin a récemment utilisé l'un de ces manuels comme point de départ de l'ouvrage qu'il a dirigé : A. Corbin, 1515 et les grandes dates de l'histoire de France revisitées par les grands historiens d'aujourd'hui, Paris, Seuil, 2005, 475 p. et A. RODES, La réception de l'histoire romaine dans l'enseignement secondaire de 1880 à nos jours, thèse de doctorat de l'université de Toulouse II-Le Mirail, en cours, sous la direction de Corinne Bonnet.

20 G. Lacour-Gayet et P. Guiraud, Histoire romaine depuis la fondation de Rome jusqu'à l'invasion des barbares, Paris, F. Alcan, 1885, p. 252.

21 A. Alba, J. IsaAc et A. Malet, Histoire romaine, à l'usage de la classe de 5e, Paris, Librairie Hachette, $7^{\mathrm{e}}$ édition, 1931 ( $1^{\text {re }}$ édition 1924), p. 181. 
"Pour faire comprendre aux écoliers ce qu'est la colonisation romaine, les auteurs la comparent à la colonisation de l'Algérie, qui, on le sait, n'est pas autre chose qu'un processus de civilisation. Ce faisant, ils feignent de ramener à un élément déjà connu (la colonisation de l'Algérie) quelque chose de nouveau, alors qu'en réalité les deux sont produits simultanément par le même texte et qu'il s'agit bien moins d'“expliquer" la colonisation romaine que de justifier la colonisation française en jouant de la commutation Gaulois/ Algériens : "Des colons Romains vinrent s'établir en Gaule, comme des Français vont de nos jours se fixer en Algérie 22 ." "

Les analogies entre le présent et le passé peuvent avoir un intérêt à condition que l'enseignant prenne appui sur les connaissances des élèves, ce qui est bien différent que de construire deux modèles distants l'un par rapport à l'autre et ignorés des élèves.

Jules Toutain participa pour sa part à de nombreux livres d'histoire aux éditions Belin. Dans ces manuels, sans reprendre les comparaisons dont il vantait les mérites dans ses travaux universitaires, il présentait la romanisation de façon très positive. Ainsi, dans un manuel de 1925, censé traiter de l'" Histoire du Moyen Âge ", il consacrait de longues pages à l'époque romaine et présentait sans nuances les bienfaits de la conquête :

"Si les Gaulois subirent facilement la domination romaine, c'est qu'elle leur parut préférable à celle des chefs qui les opprimaient ${ }^{23}$."

Il présente la conquête romaine de façon très positive, notamment parce qu'il est convaincu qu'elle a permis d'empêcher l'invasion des Germains. Une telle présentation a pu influencer la perception qu'ont les élèves de la colonisation menée par leur pays. Il faut donc s'interroger sur la portée des manuels scolaires.

\section{Quel est l'impact d'un enseignement faisant l'éloge de la colonisation?}

À partir des années 1930, Jules Toutain ne rédige plus de manuels scolaires et ses prises de position sont plus discrètes, son activité se limitant désormais à l'étude d'Alésia. Cependant, il convient de s'interroger sur l'impact d'une présentation très positive de la colonisation sur plusieurs générations d'élèves. Les manuels scolaires font depuis quelques années l'objet d'études spécifiques qui ont permis de prendre en compte leurs

22 D. Maingueneau, Les Livres d'école de la République, 1870-1914, discours et idéologie, Paris, Le Sycomore, 1979, p. 173.

23 D. Blanchet et J. Toutain, Histoire de l'Europe et particulièrement de la France depuis la fin du ve siècle jusqu'à la guerre de Cent Ans, Saint-Cloud, Belin frères, 1925, p. 19-20 : http://gallica.bnf.fr/ark :/12148/bpt6k115645x/f26.image.r=.langFR.swf (site consulté le 17/03/2011). 
limites, en particulier concernant l'impact qu'ils ont pu avoir sur la construction d'une identité commune. Pour Christian Amalvi, les manuels de l'école primaire ont sans doute joué un rôle dans l'adhésion à la colonisation, mais la remarque vaut pour ceux de collège, même s'ils concernent moins d'élèves :

"Aujourd'hui on peut légitimement se demander dans quelle mesure les livres d'école qui, tout au long de la Troisième République, glorifièrent la colonisation, n’ont pas indirectement contribué à prolonger le drame algérien ${ }^{24}$. "

Cependant, il faut garder à l'esprit les nuances apportées par Philippe Joutard, lors du même colloque intitulé Enseigner l'histoire : des manuels à la mémoire:

"Il suffit d'avoir enseigné quelques années pour savoir le fossé qui sépare l'enseignement dispensé de celui qui est reçu, mémorisé dans l'immédiat, et surtout qui subsistera dans la conscience ${ }^{25}$.»

Consciente des distances entre l'enseignement tel que l'on peut l'entrevoir dans les manuels et tel qu'il a été reçu par les élèves, il me semble cependant que l'on doit leur reconnaître une certaine influence, soulignée par Christian Goudineau. À l'occasion de la publication de l'ouvrage César et la Gaule ${ }^{26}$, en 1990, il avait effectué un sondage pour savoir quelle perception les Français avaient des Gaulois. Concernant la conquête, il révélait que $86 \%$ des sondés pensaient que la conquête de la Gaule avait été bénéfique et il n'y avait personne pour penser qu'elle ne l'avait pas été du tout. Ce spécialiste de la Gaule concluait que ces idées reçues étaient le fait de l'enseignement. Bien d'autres exemples montrent que la façon de présenter Vercingétorix comme un vainqueur à partir de 1870 a trouvé des prolongements tant dans la bande dessinée Astérix que dans certains manuels ou site officiels ${ }^{27}$.

Pour appréhender la distance entre les idées véhiculées par les résumés des manuels et l'enseignement effectivement reçu par les élèves, les cahiers d'élèves constituent des témoins particulièrement intéressants. Encore peu étudiés, ils reflètent le plus souvent les conceptions des manuels. Les effets positifs de la conquête romaine sont donc omniprésents, comme dans le cahier d'un élève parisien des années 1920, qui

24 Ch. Amalvi, "L'iconographie des manuels d'histoire et la mémoire collective : de la mémoire scolaire à la mémoire buissonnière 1871-1950 ", in H. MONIOT, Enseigner l'histoire: des manuels à la mémoire : travaux du Colloque Manuels d'histoire et mémoire collective, UER de didactique des disciplines, université de Paris 7, Paris/Berne/Francfort s/Main/Nancy/New York, P. Lang, 1984, p. 205.

25 Ph. JoutARD, cité par J. Lecuir " Manuels scolaires et mémoire historique ", in H. Moniot, Enseigner l'histoire, p. 217.

26 Ch. Goudineau, César et la Gaule, Paris, Errance, 1990, p. 17-20.

27 Le commentaire d'un document sur le site l'Histoire par l'image indique par exemple au sujet de Vercingétorix « le vainqueur d'Alésia " : http://www.histoire-image.org/site/ œuvre/analyse.php ?i=391\&d=1\&m=millet (site consulté le 6/10/2011). 
semble reprendre les mots du manuel de Jules Toutain cité ci-dessus et qui date des mêmes années : "Grace ${ }^{28}$ à la douceur de l'administration romaine. La Gaule aimat, imita ses nouveaux maitres ${ }^{29}$."

S’il y eut incontestablement adhésion des élites gauloises à la romanisation, ce propos est cependant à nuancer pour d'autres catégories de population et dans certaines zones éloignées des grandes cités. De même, l'administration est présentée de façon quelque peu angélique, les Gaulois ayant aussi dû payer un tribut au vainqueur. L'année précédente, cet élève avait reçu à peu près le même discours avec un professeur différent :

"La Gaule vaincue adopta rapidement la langue, les coutumes et la religion des

Romains. La domination romaine fut un bienfait pour la Gaule elle lui apporta l'unité et la civilisation ${ }^{30}$."

Après la Seconde Guerre mondiale, l'Antiquité occupe une place moins importante dans l'enseignement secondaire, mais les aspects positifs de la conquête des Gaules sont toujours exprimés, comme dans un cahier de 1958 :

"Assiégé et sans secours Vercingétorix se rendit à César en 52. Six plus tard il fut égorger $[s i c]$. La Gaule se romanisa et devint rapidement une des provinces les plus riches du monde romain ${ }^{31}$."

Les cahiers d'élèves attestent que cette façon de présenter la colonisation romaine, qui remonte à la fin du XIX $X^{e}$ siècle, a donc influencé plusieurs générations. Après la Seconde Guerre mondiale, l'intérêt pour l'Antiquité déclinant dans l'enseignement, cette conception n'a cependant pas été remise en cause. Elle perdure aujourd'hui encore, au moment où se pose le problème de la façon dont doit être enseignée la colonisation, notamment depuis qu'une loi en $2005^{32}$ a imposé qu'elle soit présentée

28 Les écrits des élèves sont transcrits dans leur orthographe d'origine.

29 Cahier conservé au Musée national de l'Éducation à Rouen : 3.4.11/1979.37498.2. Année 1923-1924, classe de 5e, Lycée Janson de Sailly à Paris.

30 Cahier conservé au Musée national de l'Éducation à Rouen : 3.4.11/1979.37498.1. Année 1921-1922, classe de 6e, Lycée Janson de Sailly à Paris.

31 Cahier conservé au Musée national de l'Éducation à Rouen : 3.4.11.00/2007.1879. Année 1958, classe de 5e, Haguenau.

32 L'article 4 de la loi du 23 février 2005 stipule que « Les programmes de recherche universitaire accordent à l'histoire de la présence française outre-mer, notamment en Afrique du Nord, la place qu'elle mérite. Les programmes scolaires reconnaissent en particulier le rôle positif de la présence française outre-mer, notamment en Afrique du Nord, et accordent à l'histoire et aux sacrifices des combattants de l'armée française issus de ces territoires la place éminente à laquelle ils ont droit... ". En réaction à cette loi, Claude Liauzu et Gérard Noiriel notamment lancèrent une pétition " Non à l'enseignement d'une histoire officielle ». 
de façon positive, les réactions se sont pourtant limitées à l'époque contemporaine ${ }^{33}$. Les analogies avec les populations d'Afrique du Nord ne sont plus invoquées, tout au moins plus comme au début du $\mathrm{Xx}^{\mathrm{e}}$ siècle ${ }^{34}$. De plus, alors que l'histoire de l'Afrique précoloniale est enfin prise en compte, dans l'enseignement secondaire avec les programmes entrés en vigueur en $2009^{35}$, l'histoire antique, elle, ne débute qu'avec la conquête des Gaules, comme le remarque très justement Christian Goudineau :

" [...] l'entrée dans l'histoire, la vraie, passe par l'arrivée des légions [romaines] et par les victoires qu'elles remportent ${ }^{36}$. "

Il fait ici allusion aux programmes d'histoire de collège, où la Gaule n'est jamais abordée comme telle. Si les discussions sur les programmes scolaires et le rôle de l'État sont aujourd'hui encore nombreuses, elles ne portent plus guère sur l'histoire ancienne. Jules Toutain, comme les autres auteurs de manuels de son époque, a donc durablement imprégné une image positive de la conquête romaine, dont les finalités, plus ou moins clairement exprimées au début $\mathrm{du} \mathrm{Xx}$ siècle, ne font plus vraiment sens aujourd'hui, même si elles continuent d'être utilisées.

\section{Conclusion}

Si des parallèles entre l'Antiquité romaine et la fin du XIX ${ }^{\mathrm{e}}$ siècle apparaissent clairement dans certains manuels, ceux de Jules Toutain se contentent de les suggérer en présentant la colonisation romaine de façon très positive, alors que ses écrits universitaires sont plus explicites. Ces rapprochements plus ou moins énoncés entre la colonisation romaine et la colonisation de l'Afrique du Nord, utilisés pour faire comprendre aux élèves les réalités anciennes, ont sans doute eu une influence pour faire accepter la colonisation contemporaine. Et comme l'indiquait Gustave Glotz, dans un article sur les enjeux de l'enseignement de l'histoire publié dans la revue où Jules Toutain s'est souvent exprimé, il s'agissait d' " [...] accommoder l'histoire au goût du jour, ou

33 A. RoDEs, «"Enseigner la colonisation de façon positive" ne dérange personne à condition qu'il s'agisse de la colonisation antique ", communication donnée lors du Colloque international de didactique de l'histoire, géographie et éducation à la citoyenneté, organisé à Lyon les 17 et 18 mars 2011 par l'Institut français de l'Éducation.

34 Aujourd'hui, la conquête romaine n'est plus utilisée pour faire accepter la colonisation mais pour montrer que, de part et d'autre de la Méditerranée, au-delà de la conquête arabo-musulmane, les élèves français, même lorsqu'ils sont issus de l'immigration, ont une culture commune, résultant de la conquête romaine. Ainsi, les programmes de la classe de sixième, entrés en vigueur en 2009, proposent d'étudier « [...] un exemple au choix d'une ville romaine en Gaule ou en Afrique du Nord ", Bulletin officiel, $\mathrm{n}^{\circ} 6$ spécial du 28 août 2008, p. 8.

35 Bulletin officiel, $\mathrm{n}^{\circ} 6$ spécial du 28 août 2008, p. 19.

36 Goudineau, César et la Gaule, p. 19-20. 
transposer dans le passé des passions contemporaines ${ }^{37}$ ". La volonté de Jules Toutain de transposer dans le passé ses passions contemporaines est sans doute le fait de la multiplicité de ses fonctions - parmi lesquelles celles d'archéologue impliqué dans l'organisation de l'archéologie tunisienne au service de l'état colonisateur, spécialiste de la province romaine de Tunisie et auteur de manuels d'histoire antique et contemporaine, dans le premier tiers du $\mathrm{XX}^{\mathrm{e}}$ siècle - plus qu'une volonté délibérée de manipulation politique.

Aurélie Rodes

Doctorante en histoire ancienne

à l'Université de Toulouse (UTM)

64 rue Adolphe Coll

F-31300 Toulouse

aurelie.rodes@ac-toulouse.fr

37 G. Glotz, "Réflexions sur le but et la méthode de l'histoire ", Revue internationale de l'enseignement 54 (1907), p. 481. 